\title{
Utilisation de paramètres bioécologiques pour analyser la biodiversité ichtyologique de l'Aire Marine Protégée (AMP) de Joal-Fadiouthau Sénégal et sa périphérie non protégée à la pêche
}

\author{
Serigne Modou Sarr, \\ Institut Supérieur de Formation Agricole et Rurale ex ENCR, \\ Université de Thiès, Bambey, Sénégal \\ Modou Thiaw, \\ Institut Sénégalais de Recherches Agricoles, \\ Centre de Recherche Océanographique Dakar-Thiaroye, Sénégal \\ Balla Aramane Mbengue, \\ Ministère de l'économie Maritime, Sénégal
}

Doi: 10.19044/esj.2018.v14n33p349 URL:http://dx.doi.org/10.19044/esj.2018.v14n33p349

\begin{abstract}
With global changes, concerns about marine ecosystems and population dynamics are of increasing interest to researchers. As such, knowledge of the specific biodiversity of exploited marine ecosystems is generally considered a key factor in their resilience. This paper is a comparative study that focuses on analyzing the bioecological effects of MPA Joal-Fadiouth on fish populations by comparing the inside $v s$. outside of the MPA. Data were collected from scientific surveys conducted in cold and hot seasons in 2015. In each survey, 16 stations were sampled; 9 inside the MPA and 7 outside. The MPA is in open Ocean and the analysis of environmental parameters shows that the MPA area is a homogeneous environment. The variability is at the seasonal level. This variability is similar inside and outside the MPA. A total of 67 species in 37 families were found in the reserve, against 41 species in 32 families outside MPA. The biological indicators analysis shows a similar species richness and biomass between the two areas. However, total abundance is higher in the protected area. On the other hand, larger fish are more likely to be outside the protected area. In the MPA, 20 species sampled are in spawning period, but over $80 \%$ of individuals are immature. The Joal-Fadiouth MPA implementation significantly improves the conservation of biodiversity with a dominance of herbivorous species and lower trophic levels. In addition, the reserve is a nursery area for many species (sardines, octopus, and shrimp) and reproduction for some species
\end{abstract}


characteristics of the area, e.g.Hemiramphus brasiliensis and Stephanolepis hispidus.

Keywords: Marine protected areas, biodiversity, bioecology, Evaluation, indicators, Senegal

\section{Résumé}

Avec les changements globaux, les préoccupations sur les écosystèmes marins et la dynamique des peuplements intéressent de plus en plus les chercheurs. À ce titre, la connaissance de la biodiversité spécifique des écosystèmes marins exploités est généralement considérée comme un facteur clé de leur résilience. Cette étude comparative a donc pour objectif d'analyser les effets bioécologiques de l'AMP de Joal-Fadiouth sur les peuplements de poissons. Des pêches expérimentales sont réalisées en 2015 , en saisons froide et chaude. A chaque campagne, 16 stations sont échantillonnées dont 9 stations dans l'AMP et 7 hors des limites de l'AMP. L'analyse des paramètres environnementaux montre que le milieu est homogène et il est surtout caractérisé par une différence saisonnière entre l'AMP et hors de l'AMP. Au total, 67 espèces réparties dans 37 familles sont capturées dans l'aire protégée contre 41 espèces réparties dans 32 familles hors de l'AMP. L'analyse des indicateurs biologiques montre une richesse spécifique et une biomasse similaires entre les deux zones. Cependant, l'abondance totale est plus élevée dans l'aire protégée. Par contre, les poissons de grande taille sont en proportion plus nombreux en dehors de la zone protégée. Dans l'AMP, 20 espèces échantillonnées sont en période de reproduction, mais plus de $80 \%$ des individus sont immatures. La mise en réserve améliore sensiblement la conservation de la biodiversité avec une dominance d'espèces herbivores et de bas niveaux trophiques. De plus, c'est une zone de nourricerie pour plusieurs espèces (sardinelles, poulpe, crevettes) et de reproduction pour certaines espèces caractéristiques de la zone, e.g. Hemiramphus brasiliensis et Stephanolepis hispidus.

Mots-clés: Aires marines protégées, biodiversité, bioécologie, évaluation, indicateurs, Sénégal

\section{Introduction}

Au Sénégal, en l'espace d'un quart de siècle, l'effort de pêche a été multiplié par 2,5 entraînant une exploitation excessive des ressources halieutiques (Gascuel et al., 2002), laquelle serait à l'origine de la surexploitation des principales ressources démersales côtières. La biomasse de ces stocks a connu une nette diminution, pouvant aller de 50 à $90 \%$ de leur 
biomasse à l'état vierge (Gascuel et al., 2002 ; Thiaw et al., 2009; Thiao et al., 2012 ; Ndiaye et al., 2013).

Pour une gestion durable des ressources halieutiques, le gouvernement du Sénégal a pris une série de mesures, notamment le gel de l'octroi de toute licence de pêche démersale côtière, la réduction de l'effort de pêche des chalutiers étrangers entre autres (CRODT, 1991). Cependant, ces mesures n'ont pas abouti à inverser la tendance à la baisse de la biomasse des espèces halieutiques (Fall, 2009). Malgré le nombre élevé d'AMP créées, une revue de la littérature scientifique montre que des points de vue opposés demeurent sur l'efficacité des aires marines protégées (Hilborn, 2014). D'une part, les effets positifs de certaines AMP au plan bioécologique sont confirmés par de nombreux auteurs (Mesnildrey et al., 2010 ; Albaret et al., 2005). D'autre part, la réalité des mesures de protection est parfois controversée par des scientifiques (Samoilys et al., 2007). De plus, les résultats négatifs trouvés sont souvent moins publiés que les résultats positifs et les impacts observés pour un cas donné ne sont pas forcément reproductibles ailleurs (Claudet, 2010). Les effets attendus peuvent par conséquent être positifs, nuls voire même négatifs par rapport aux objectifs attendus.

Depuis la mise en place de l'AMP de Joal-Fadiouth, deux études bioécologiques ont été réalisées (Thiao \& Mbaye, 2012). Pourtant, les études faites par Sarré et al. (2009) ne concernent que la saison froide et l'engin utilisé (une senne de plage) ne permet pas l'échantillonnage à des profondeurs de plus de $2 \mathrm{~m}$. Nos travaux constituent alors une étude bioécologique complémentaire et ils ont permis de démontrer l'efficacité de la mise en réserve de l'AMP de Joal Fadiouth sur les peuplements ichtyologiques.

\section{Matériel et méthodes \\ Le cadre de l'étude}

L'AMP de Joal-Fadiouth se situe dans la commune du même nom au Sénégal. Localisée dans la partie méridionale du département de Mbour, sur la Petite Côte, à $112 \mathrm{~km}$ de Dakar, la commune de Joal-Fadiouth se situe entre $14^{\circ} 06^{\prime} 01^{\prime}$ et $13^{\circ} 13^{\prime} 00^{\prime}$ de latitude Nord et $16^{\circ} 47^{\prime} 07^{\prime}$ et $16^{\circ} 53^{\prime} 05^{\prime} \mathrm{de}$ longitude Ouest (Figure 1). Sa superficie est de $174 \mathrm{~km}^{2}$ (17 $400 \mathrm{ha}$ ). Les limites de l'AMP comprennent les dépendances maritimes de la commune, un bras de mer ainsi qu'un important réseau de mangroves. La zone protégée comprend les dépendances maritimes de la commune de Joal-Fadiouth, un bras de mer, ainsi, qu'un important réseau de mangroves (Figure 1). Les moyennes mensuelles de température les plus élevées sont supérieures à $30^{\circ} \mathrm{C}$ et elles sont enregistrées entre les mois d'avril et de juin. Les plus basses sont de l'ordre de $25^{\circ} \mathrm{C}$ et elles sont enregistrées entre les mois de décembre et de janvier. Les levées bathymétriques obtenues par Sarré et al. (2009) révèlent 
l'existence d'un plateau assez large, avec des profondeurs relativement faibles de l'ordre de 3 à $5 \mathrm{~m}$, sur l'ensemble de la zone balayée.

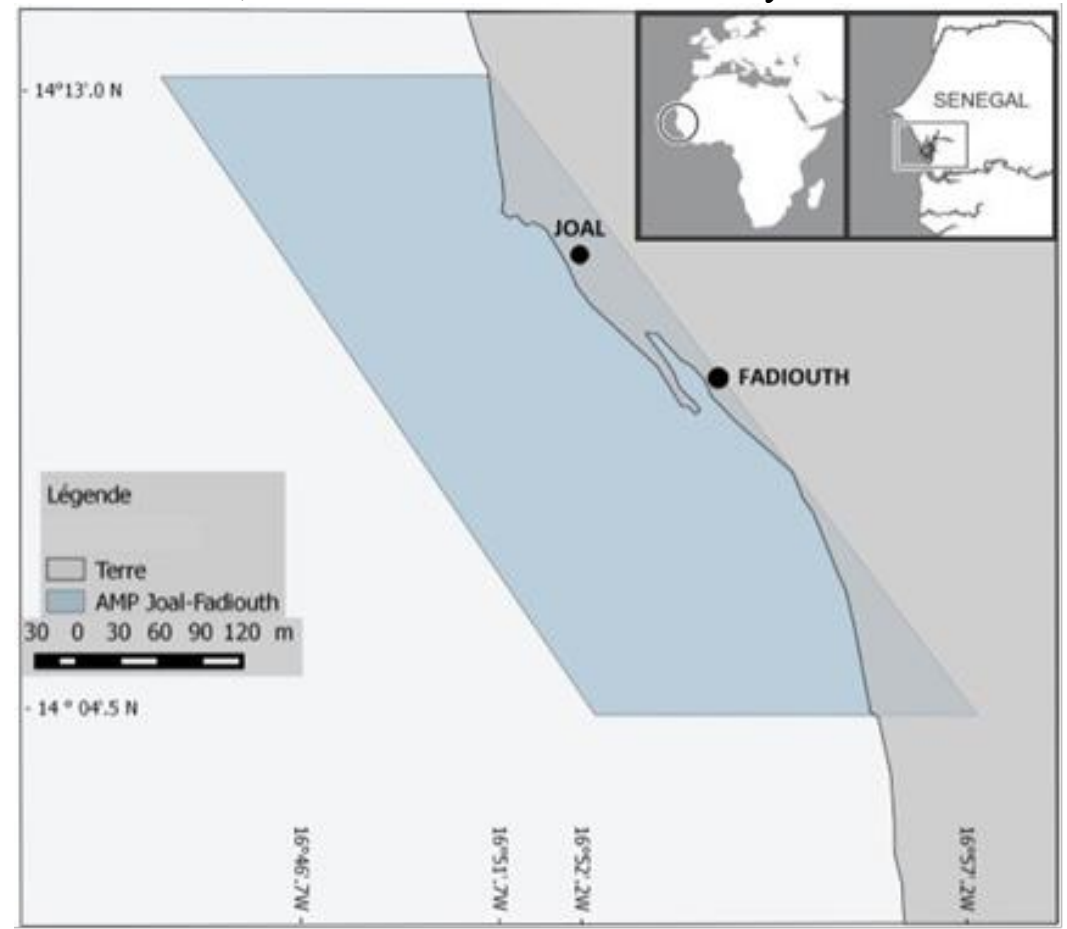

Figure 9. Localisation et situation géographique de l'AMP de Joal-Fadiouth.

\section{Echantillonnage}

L'échantillonnage a été réalisé en deux (2) campagnes de pêche pendant les mois d'avril et de septembre correspondant aux deux grandes saisons hydro-climatiques du milieu, à savoir la saison froide et la saison chaude (Diallo et al., 2008). L'échantillonnage aléatoire a été adopté pendant les pêches expérimentales en subdivisant la zone d'étude en deux sousensembles : dans l'AMP et en dehors de l'AMP. Dans l'AMP, neuf(09) coups de pêche ont été réalisés contre sept (07) hors de l'AMP. Chaque coup de pêche, sur une surface bien déterminée ( $0,5 \mathrm{ha})$, correspond à une station de pêche. La distance entre chaque station située hors de la réserve et les limites de l'AMP est au minimum d'un (01) kilomètre afin d'éviter la capture des individus se déplaçant de l'intérieur vers l'extérieur.

La senne tournante coulissante a été utilisée lors de ces pêches expérimentales. D'une longueur totale de $250 \mathrm{~m}$, une hauteur de chute de 20 $\mathrm{m}$ et des mailles de $14 \mathrm{~mm}$, la senne utilisée balaie une surface estimée à 0,5 ha par coup de pêche de $30 \mathrm{mn}$. Les relevés effectués en simultané avec la pêche (Tableau 1) sont la profondeur (sondeur à main), la salinité (réfractomètre de précision à 1 unité de salinité), la teneur en oxygène et la température (sonde multi-paramètre, YSI 85) et la transparence ou turbidité 
(disque de Secchi, diamètre 10-15 cm). Les paramètres physico-chimiques (salinité, teneur en oxygène et température) ont été mesurés en surface et au fond. Les poissons récoltés ont été triés par espèce, puis les individus de l'ensemble ou d'une fraction importante (30 individus au maximum) ont été pesés (poids total frais, au gramme près) et mesurés au millimètre près. La longueur relevée est la longueur totale $\left(\mathrm{L}_{t}\right)$ mesurée du point le plus en avant de la tête, bouche fermée, jusqu'à l'extrémité de la queue, la queue étant rabattue (Badts \& Bertand, 2012).

Tableau 1. Variables mesurées lors des pêches expérimentales.

\begin{tabular}{|l|l|l|l|}
\hline $\begin{array}{l}\text { Variables du plan } \\
\text { d'échantillonnage }\end{array}$ & \multicolumn{1}{|c|}{ Variables du milieu } & $\begin{array}{c}\text { Variables de } \\
\text { la population }\end{array}$ & \multicolumn{1}{|c|}{$\begin{array}{c}\text { Variables de } \\
\text { l'individu }\end{array}$} \\
\hline Campagne & Profondeur & Espèce \\
Coup de pêche & Transparence & Taille \\
Lieu de pêche & Salinité de surface & Poids pêché & Classes de maturité \\
Pate & Poids \\
Mois & Température de surface & & \\
Heure de début & Température de fond & & \\
Heure de fin & Oxygène de surface & & \\
Position & Oxygène de fond & & \\
\hline
\end{tabular}

\section{Paramètres bioécologiques}

Dans cette étude, les indicateurs suivants ont été utilisés :

- Richesse spécifique totale et moyenne

La richesse spécifique totale correspond au nombre d'espèces rencontrées lors de l'ensemble des comptages d'une zone. La richesse spécifique moyenne correspond à la moyenne du nombre d'espèces rencontrées dans une zone.

- Occurrence des espèces de poissons $(\mathrm{Fo})$

La fréquence d'occurrence $\mathrm{F}_{\circ}$ d'une espèce $i$ correspond au pourcentage de relevés pour lesquels l'espèce est rencontrée. Une espèce pourra être qualifiée de permanente (75-100\%), fréquente $(50-75 \%)$, occasionnelle $(25-50 \%)$ ou rare $(0-25 \%)$ en fonction de sa fréquence d'occurrence (Tessier, 2005).

$F_{0}=\frac{E_{i}}{E_{t}}$ où :

$\mathrm{E}_{\mathrm{i}}$ : le nombre de coups de pêche où l'espèce est présente et $\mathrm{E}_{\mathrm{t}}$ : le nombre total de coups.

- Abondance spécifique (A)

L'abondance est calculée en sommant d'abord les abondances des coups de pêche. Le facteur multiplicatif « 4 »s'explique par le fait que le 
coefficient d'échappement de la senne tournante est de $50 \%$, l'abondance multipliée par 2 pour avoir $100 \%$ de la capture. Selon Tessier (2005), l'abondance a été calculée selon la formule suivante :

$A=\frac{\sum_{\mathrm{i}} N_{\mathrm{i}}}{} * 4$ où :

$\mathrm{Ni}$ : nombre total d'individu de l'espèce $i$;

$\mathrm{x}$ : nombre total de coups de pêches réalisés.

- Biomasse spécifique (B)

L'estimation de la biomasse est réalisée en faisant la même procédure pour calculer l'abondance. Dans ce cas, la formule est la suivante selon McClanahan \& Mangi, (2004):

$B=\frac{\sum B_{i}}{x} * 4$

où :

Bi : biomasse totale de l'espèce $i$.

- Niveau trophique moyen (TL)

Les niveaux trophiques moyens des espèces sont pondérés par les biomasses selon la formule (McClanahan \& Mangi, 2004):

$\mathrm{TL}=\frac{\sum_{\mathrm{i}=1}^{\mathrm{N}} \mathrm{b}_{\mathrm{i}}{ }^{*} \mathrm{TL}}{\sum \mathrm{b}_{\mathrm{i}}}$ où :

$\mathrm{b}_{\mathrm{i}}$ : biomasse de l'espèce $i$,

$\mathrm{TL}_{\mathrm{i}}$ : niveau trophique moyen de l'espèce $i$

$\mathrm{N}$ : le nombre total d'espèces.

\section{Analyse du spectre de taille}

Les indicateurs basés sur les spectres de tailles sont donc potentiellement utilisables pour comprendre les effets de la pêche et la dynamique des peuplements exploités (Shin et al., 2005).

\section{Analyses statistiques}

L'environnement aquatique de l'AMP de Joal-Fadiouth a été décrit par une analyse descriptive univariée (boxplots), une analyse bivariée (analyse de variance à un facteur, le test-U non paramétrique de Wilcoxon (Mann et Whitney) et une analyse multivariée (ACP: Analyse en Composantes 
Principales). Les différents indicateurs choisis ont été analysés en comparant les paramètres de position (Minimum, Maximum et Moyenne) et de dispersion (Ecart-Type) à l'échelle spatiale. Les valeurs moyennes des paramètres mesurés ont été comparées par le test non-paramétrique de Wilcoxon (MannWhitney) au seuil $\alpha$ de $5 \%$. Pour faciliter l'interprétation des tests, nous avons illustré les résultats obtenus avec des boxplots.

La structure des communautés de poissons a été étudiée par une Analyse Factorielle des Correspondances (AFC) appliquée sur la matrice (abondances des espèces * stations). Cette matrice a été triée pour ne retenir que les espèces ayant une occurrence supérieure à $25 \%$ dans au moins une des deux zones (AMP ou Hors AMP).

Les longueurs totales des poissons, utilisées pour étudier les structures en tailles, sont regroupées en classes définies par des valeurs centrales. Le nombre d'individus par classe de tailles a été transformé par la fonction logarithme $\ln (\mathrm{x}+1)$ dans les spectres de taille.

Les traitements statistiques et les graphiques ont été réalisés à l'aide du logiciel statistique ' $R$ ' disponible en accès libre sur internet (R Development Core Team, 2015).

\section{Résultats}

\section{Caractérisation de l'environnement}

L'analyse des paramètres physico-chimiques enregistrés (température, salinité et oxygène) ne montre pas une différence significative entre les valeurs mesurées à la surface et au fond (test de Wilcoxon, p-value > 0,5). Par conséquent, seules les valeurs de surface sont retenues dans la suite de cette étude. De même, aucune différence significative n'est observée concernant une variabilité spatiale de la température, de la salinité et de l'oxygène (Figure 2) entre l'AMP et la zone hors de l'AMP ( p-value > 0,5).Pourtant, la transparence et la profondeur sont significativement différentes entre les deux zones ( $\mathrm{p}$-values $<0,05)$.

a) Profondeur (m)

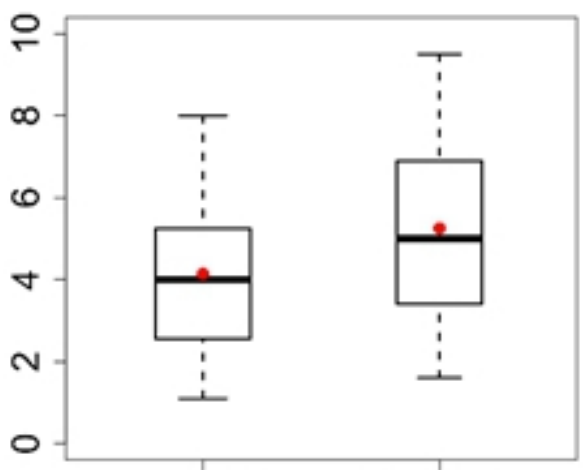

Froide
Chaude b) Transparence (m)

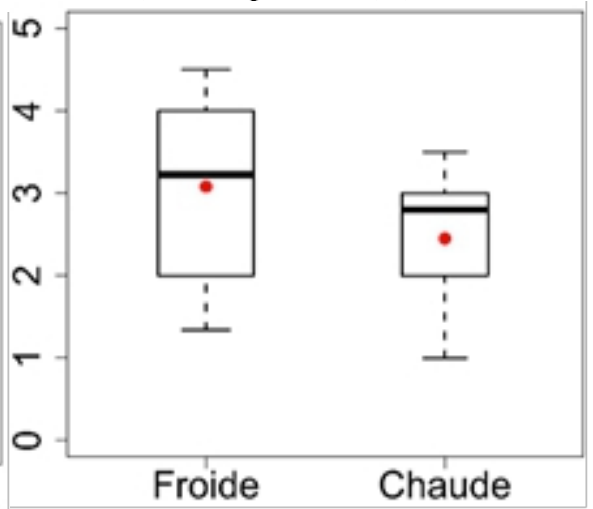


c) Température $\left({ }^{\circ} \mathrm{C}\right)$

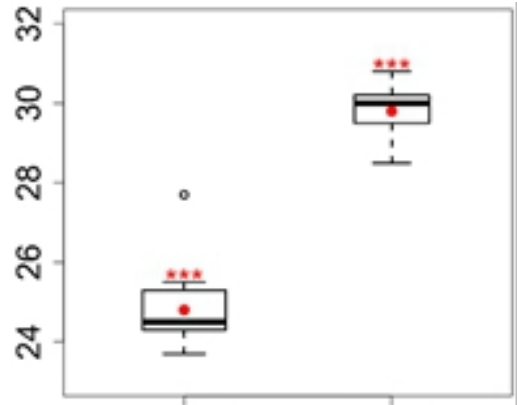

Froide Chaude d) Salinité (PSU)

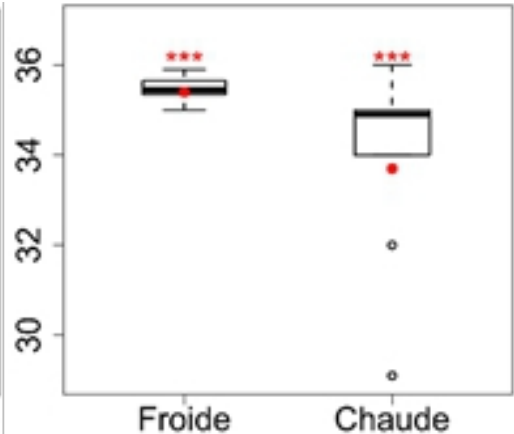

Froide

Chaude

e) Oxygène $(\%)$

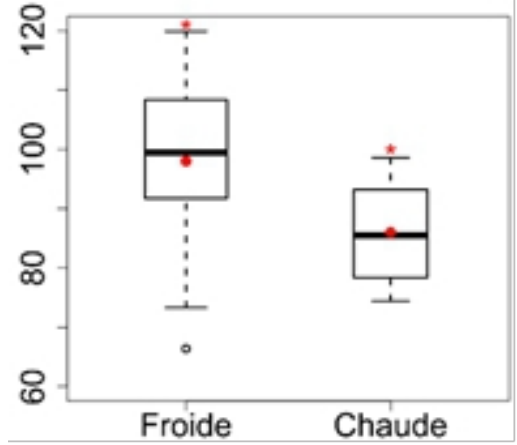

Figure 2. Boxplots par saison des variables de l'environnement (valeurs de surface pour salinité, température et oxygène dissous).

L'histogramme des valeurs propres de l'ACP centrée et réduite (Figure 3) suggère de retenir les deux premiers axes (Axe $1: 55,45 \%$ et Axe $2: 19,50$ $\%)$. Le plan factoriel 1-2 explique environ 74,95\% de l'inertie totale. L'axe 1 oppose la température avec les autres paramètres mesurés (l'oxygène, la salinité, la profondeur et la transparence). En effet, ceci confirme la température élevée pendant la saison chaude. L'axe 2 oppose l'oxygène et la salinité ensemble contre la profondeur et la température de l'autre côté. La différence entre l'AMP et hors de l'AMP est surtout en relation avec la profondeur relevée dans la zone (Figure 4).

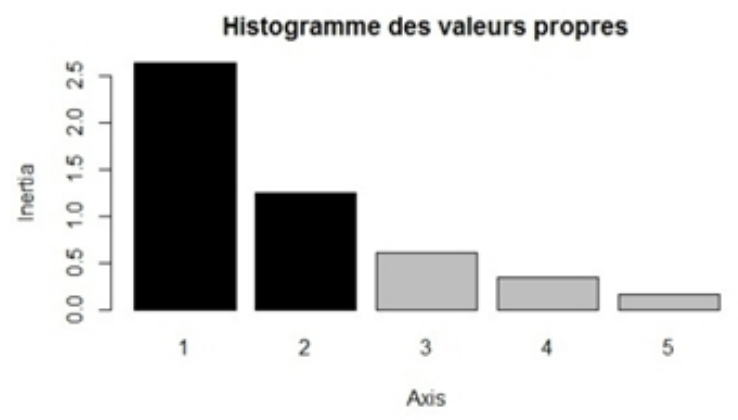

Figure 3. Histogramme des valeurs propres 


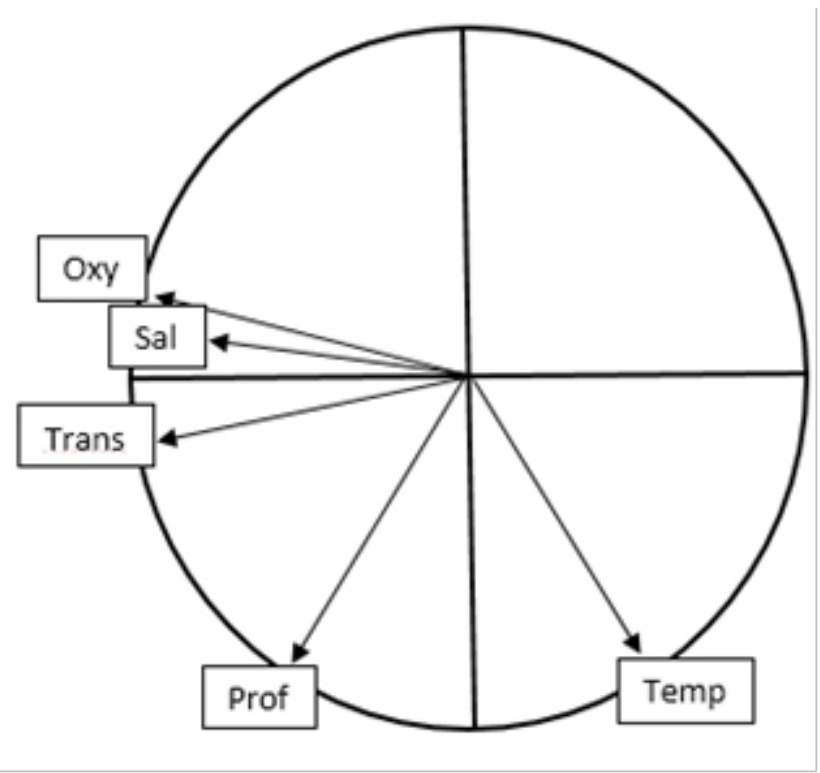

Figure 4. Analyse en Composantes Principales

\section{Inventaire des espèces dans les dans deux zones}

$\mathrm{Au}$ total, 78 espèces appartenant à 45 familles sont observées dans l'AMP de Joal-Fadiouth et hors de l'AMP. Les familles de poisson les plus représentées, en nombre d'espèces, sont les Carangidae et les Sparidae (6 espèces chacune). Ces deux familles sont suivies par les Haemulidae et les Mugilidae (5 espèces chacune), les Tetraodontidae (4 espèces) et les Sciaenidae et les Soleidae (3 espèces chacune). Les Cichlidae, les Clupeidae, les Gerreidae, les Serranidae sont représentées par deux espèces chacune. Enfin, les autres familles sont composées d'une seule espèce. Des crustacés (Portunidae avec 3 espèces) et des gastéropodes (Volutidae, Muricidae ; 2 espèces chacune) sont aussi recensées dans cette étude.

\section{Occurrence, abondance et biomasse}

En termes d'occurrence, huit (8) espèces sont recensées dans plus de $50 \%$ des stations étudiées. Il s'agit d'abord des deux espèces permanentes dans le milieu (Hemiramphus brasiliensis et Stephanolepis hispidus). Les Hemiramphus brasiliensis et Stephanolepis hispidus sont rencontrées dans toutes les stations hors de l'AMP et dans plus de $66 \%$ des stations de l'AMP. D'autres espèces fréquentes telles que Nicholsina usta $(68,75 \%)$, Sepia officinalis (75 \%), Ablennes hians (56,25\%), Pseudupeneus prayensis (62,5 $\%)$, Mugil bananensis (56,25\%) et Pagrus caeruleostictus (56,25\%) sont aussi recensées dans la zone d'étude. Diverses espèces sont récurrentes dans une zone seulement comme Ephippion guttifer hors de l'AMP et les espèces de petite taille (Sardinella maderensis, Eucinostomus melanopterus, Diplodus 
bellottii et Pomadasys incisus) dans la réserve. Eucinostomus melanopterus est la plus abondante dans la zone d'étude. Elle représente à elle seule 25,45 $\%$ de l'abondance totale. Les espèces Sardinella maderensis (40,08\%) et $M$. bananensis $(8,21 \%)$ dominent en proportion d'abondance hors de l'AMP. Par contre, dans la réserve, le peuplement est dominé par E. melanopterus $(29,75$ $\%$ ) et les deux espèces de Mugilidae (Liza dumerili et M. bananensis) qui représentent ensemble 28,84\% du nombre total des individus capturés. L'abondance élevée dans l'AMP des deux Cichlidae (Sarotherodon melanotheron et Tilapia guineensis) est à signaler.

En termes de biomasse, $M$. bananensis est la plus abondante avec 17,9 $\%$ de la biomasse totale de la zone d'étude. Hors de l'AMP, les espèces ( $E$. guttifer, M. bananensis et S. maderensis) représentent plus de $50 \%$ de la biomasse calculée. Cependant, les proportions de biomasse de M. bananensis, E. melanopterus, Liza dumerili et des deux Cichlidae (T. guineensis et $S$. melanotheron) sont plus élevées dans l'AMP comparées aux autres espèces.

\section{Indicateurs bioécologiques}

Le peuplement de poisson, observé dans la réserve, a une richesse spécifique totale de 67 espèces contre 41 espèces dans la zone non protégée. Toutefois, 30 espèces, soit 38,5\% de l'effectif total (78 espèces) sont communes aux deux zones.

Le niveau trophique moyen par coup de pêche est en moyenne de 3,20 $\pm 0,33$ dans l'AMP et de 3,24 $\pm 0,18$ hors de l'AMP.

\section{Structure du peuplement ichtyologique}

L'analyse factorielle par correspondance réalisée sur la zone d'étude montre un plan 1-2 expliquant 29,05\% de l'inertie totale (axe $1=17,50 \%$ et axe $2=10,55 \%$ ). L'AFC permet d'isoler deux groupes : (i) un groupe d'espèces prépondérantes dans les stations hors de l'AMP et (ii) un groupe d'espèces communes aux stations des deux zones. L'AFC montre une biodiversité importante de l'AMP avec la presque totalité des espèces présente dans la réserve mis à part certaines espèces comme Diplodus senegalensis.

Le peuplement dans la zone hors de la réserve est principalement formé d'espèces de catégories écologiques marines ayant des niveaux trophiques élevés (prédateurs de premier ordre à prédominance benthophages, prédateurs de deuxième ordre généralistes et des prédateurs de deuxième ordre à prédominance piscivores) (Figures5a et 5b). Les espèces caractéristiques de ce peuplement sont Sphyraena afra (ME, p2-pi), Priacanthus arenatus (Mo, p2-ge), Balistes punctatus (Ma, p1-bt) et Diplodus senegalensis (Mo, p1-bt).

L'AMP comprend principalement des espèces estuariennes strictes $(\mathrm{Em})$ et des espèces à affinité marine $(\mathrm{ME})$. D'autres poissons d'origine marine (Mo et Ma) sont caractéristiques de la zone et ils sont pour la plupart 
de bas niveau trophique. Il s'agit de Mugil bananensis (ME, he-de), de Liza falcipinis (Em, he-de) et de Sarotherodon melanotheron (Es, he-ph). Or, les prédateurs sont aussi présents dans la réserve, i.e. Pomadasys incisus (Ma, p1bt), Diplodussargus cadenati (Mo, p1-bt), Pomadasys jubelini (Em, p1-bt), Epinephelus aeneus (ME, p2-pi), Diplodussargus cadenati (Mo, p1-bt) et Galeoides decadactylus (ME, p2-ge) (Figures, 6a et 6b).

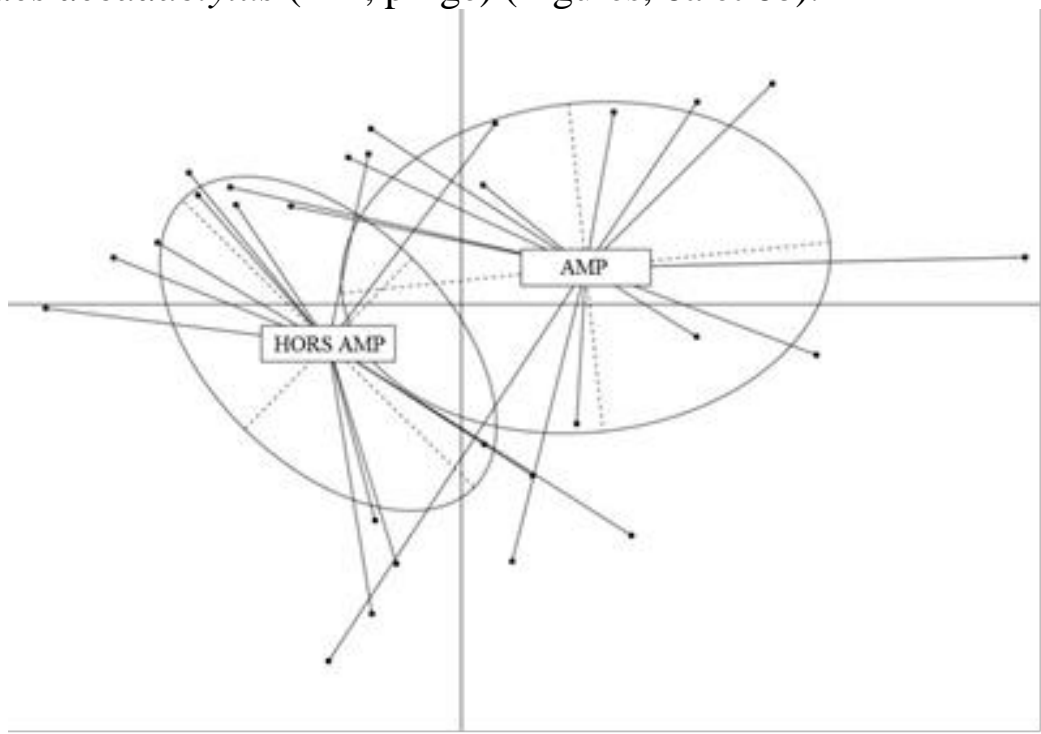

Figure 5a. Analyse en Composantes Principales, Plan factoriel 1-2. (a) - Position moyennes

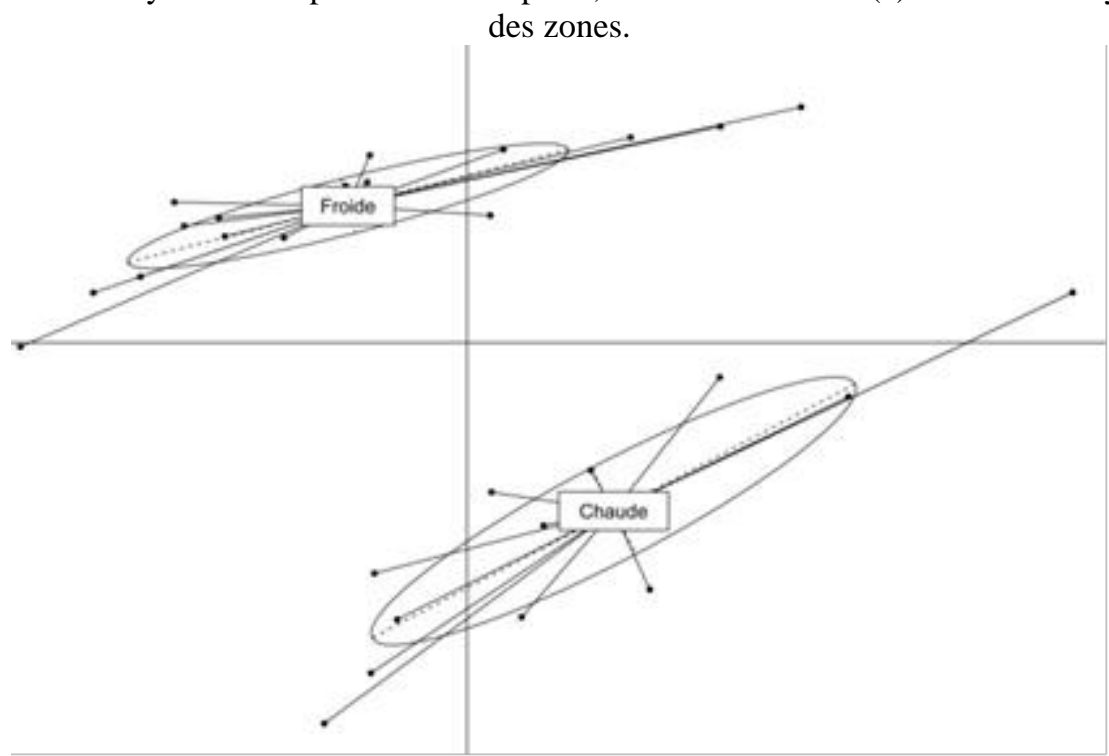

Figure 5b. Analyse en Composantes Principales, Plan factoriel 1-2. (b) - Position moyennes des saisons. 


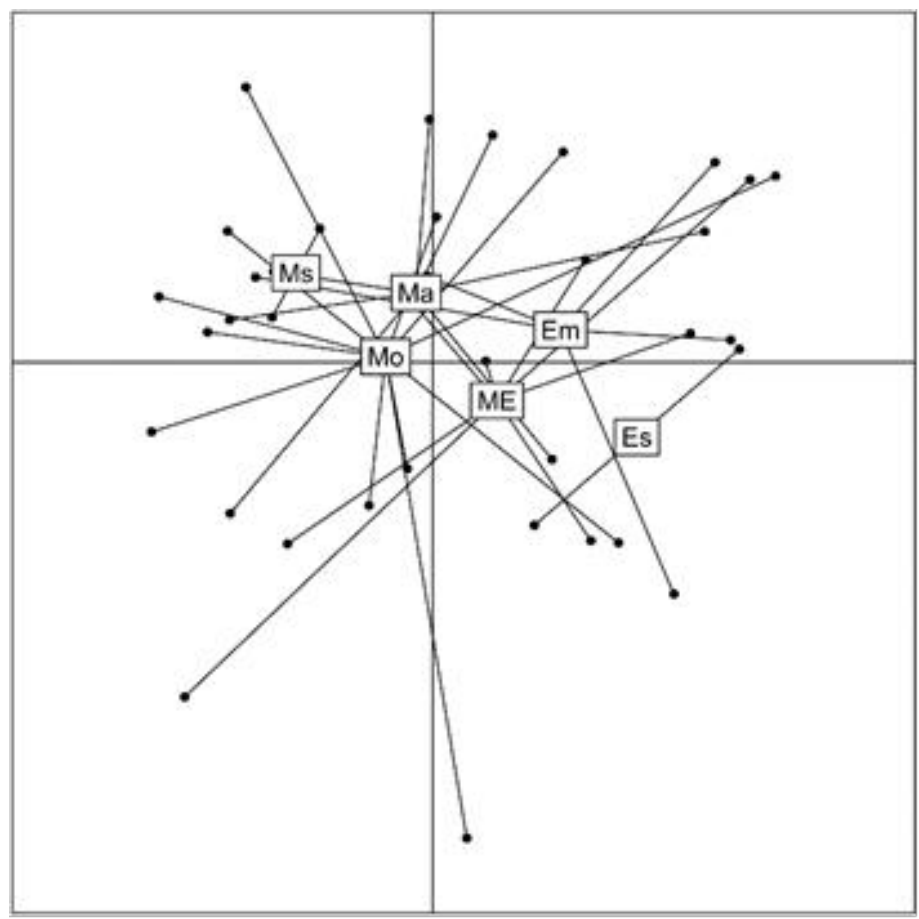

Figure 6a. Analyse Factorielle des Correspondances des peuplements - Plan factoriel 1-2. (a) - Projection des points moyens par catégories écologiques

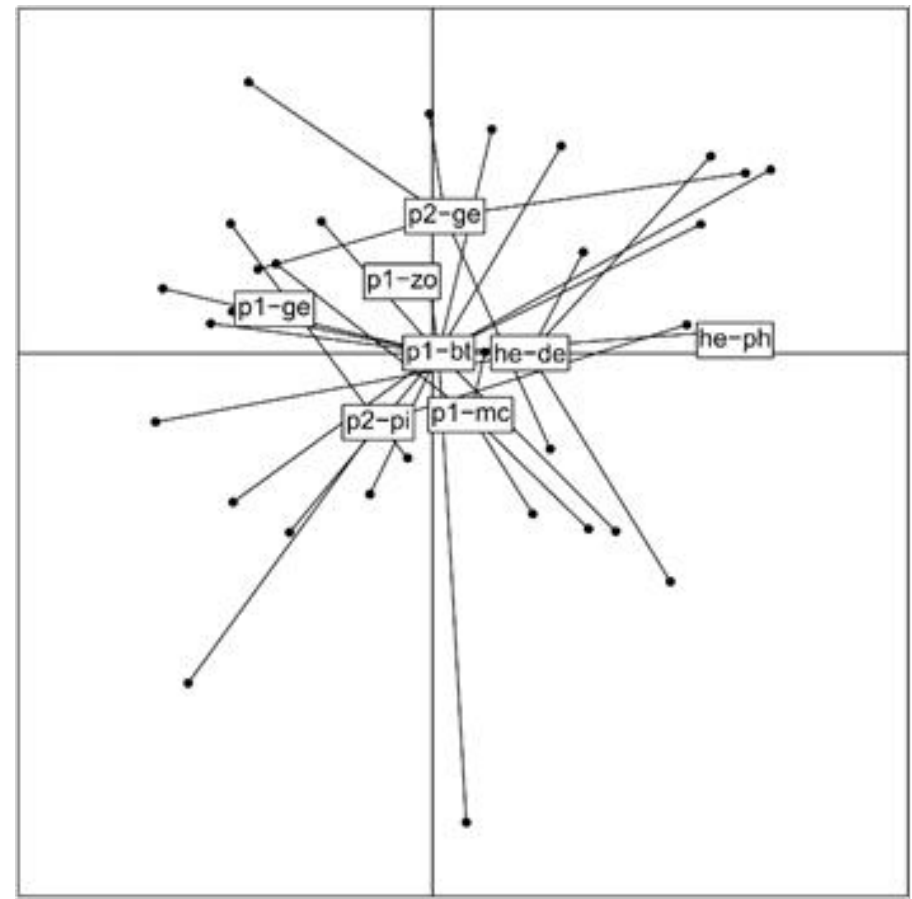

Figure 6b. Analyse Factorielle des Correspondances des peuplements - Plan factoriel 1-2. (b) - Projection des points moyens par catégories trophiques. 


\section{Spectre de taille}

La taille minimale observée chez les poissons est de $20 \mathrm{~mm}$ (Diplodus bellottii) dans l'AMP et de $60 \mathrm{~mm}$ (Priacanthus arenatus) hors de la zone protégée. Le plus grand individu de poisson mesuré a une longueur de 1080 $\mathrm{mm}$ dans la réserve contre $774 \mathrm{~mm}$ hors de la réserve pour la même espèce (Sphyraena afra). La différence de taille moyenne entre les deux zones n'est pas significative (t-test, $\mathrm{p}$-value $=0,373>0,05)$. La taille moyenne des poissons hors de l'AMP $(165,83 \mathrm{~mm} \pm 100,6)$ est de même ordre de grandeur qu'hors de la réserve $(159,57 \mathrm{~mm} \pm 86,81)$.

La figure 7 représente la distribution des fréquences de taille dans les deux zones. Le mode est pratiquement le même dans les deux zones de l'AMP. Dans la partie croissante des courbes, les tailles mesurées dans les deux zones sont globalement similaires suivant les classes modales. Cependant, à partir du mode (environ $160 \mathrm{~mm}$ ) jusqu'à une taille proche de $300 \mathrm{~mm}$, les effectifs observés dans l'AMP sont plus élevés. Enfin, au-delà de 300 mm, l'inverse est observé. En effet, le nombre d'individus de grandes tailles est plus élevé hors de l'AMP.

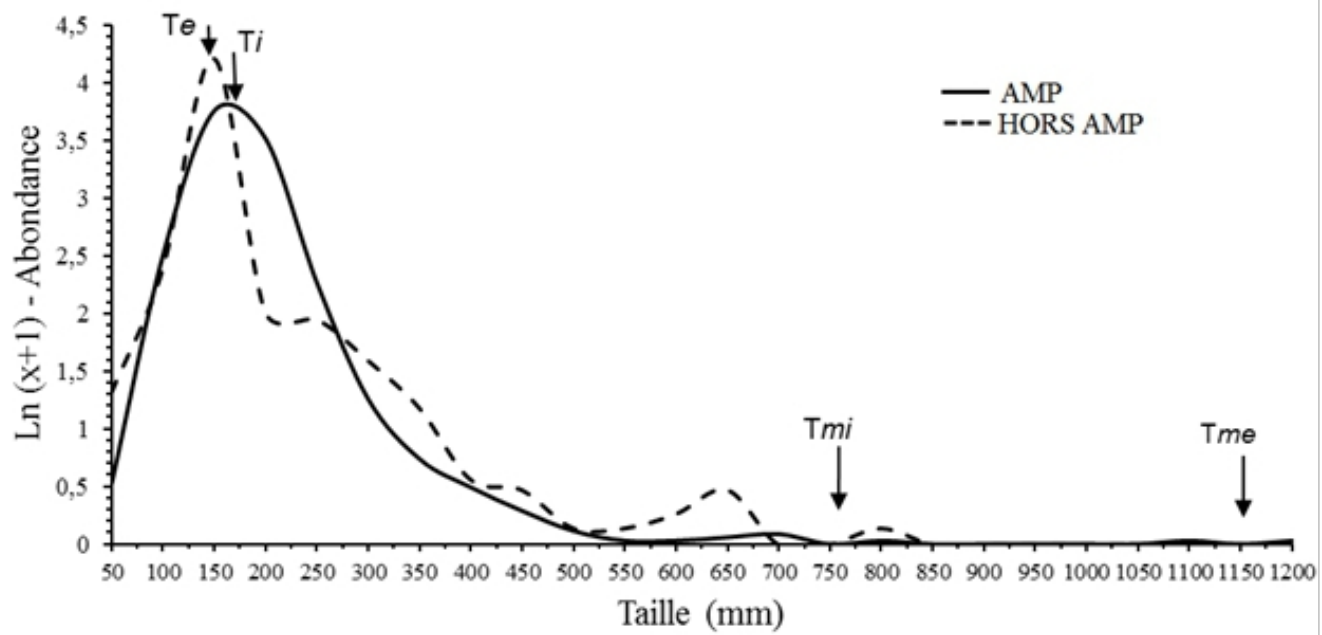

Figure 7. Spectres de taille dans l'AMP et hors de l'AMP. Tmi: Taille maximale à

l'intérieur; Tme: Taille maximale à l'extérieur; T $i$ : Taille moyenne à l'intérieur ; Te: Taille moyenne à l'extérieur.

\section{Discussions}

Les paramètres physico-chimiques (profondeur, transparence, température, salinité et teneur en oxygène) sont comparables à celles trouvées par Sarré et al. (2009) lors de l'état de référence de l'AMP de Joal-Fadiouth. De même, Thiam et al. (2012) ont aussi obtenu les mêmes résultats au niveau des zones potentielles pour l'implantation de récifs artificiels à Ngaparou (Petite Côte du Sénégal). Les gammes de températures $\left(24{ }^{\circ} \mathrm{C}-31{ }^{\circ} \mathrm{C}\right)$, d'oxygène $(65 \%-121 \%)$ et de salinité (29 PSU - 36 PSU) obtenues sont 
optimales pour la survie de diverses espèces de poissons vivant dans les zones marines et estuariennes d'Afrique de l'Ouest (Diouf et al., 2008). Ainsi, l'AMP de Joal-Fadiouth bénéficie des conditions environnementales favorables au développement d'espèces ichtyologiques.

D’après Morris (2006), la structure spatiale du peuplement peut être influencée par des paramètres physico-chimiques. Or, la variable environnementale ayant un impact sur la répartition des peuplements de poisson est la profondeur. Cependant, la profondeur est relativement faible dans la zone d'étude. De même, les fluctuations ainsi observées sur la profondeur peuvent être dues à la méthode de relevé des données (profondimètre tenu par la main) et/ou aux mouvements de l'eau. Pour les autres paramètres relevés (salinité, température et pourcentage de saturation en oxygène), les valeurs notées entre l'AMP et hors de l'AMP sont comparables. Enfin, la proximité des points moyens des deux zones (stations) au niveau de l'ACP conforte l'hypothèse d'un milieu relativement homogène.

En somme, les deux zones apparaissent comme des milieux homogènes du point de vue environnemental. La seule différence réelle enregistrée se situe au niveau saisonnier. Les éventuelles différences de structure et d'organisation des peuplements ne sauraient ainsi être considérées comme une conséquence des changements des paramètres physico-chimiques.

Une analyse globale de la richesse spécifique totale montre que soixante-sept (67) espèces sont rencontrées dans l'AMP alors que Sarré et al. (2009) avaient trouvé 56 espèces. La richesse spécifique totale de l'AMP de Joal-Fadiouth est aussi relativement élevée par rapport à l'AMP de Cayar avec 26 espèces relevées (Ndiaye, 2014). Plusieurs facteurs comme le type d'engin utilisé, la technique d'échantillonnage, le nombre de coups de pêche réalisé ou les différentes saisons climatiques échantillonnées pourraient expliquer le plus grand nombre d'espèces rencontrées dans la présente étude. En effet, d'après Lobry (2003), la probabilité de capturer une espèce supplémentaire augmente avec la surface explorée et le nombre de prélèvements effectués.

Huit espèces peuvent être qualifiées au moins de fréquentes (présentes sur au moins $50 \%$ des stations). Deux espèces, en l'occurrence Hemiramphus brasiliensis et Stephanolepis hispidus, se présentent comme permanentes dans la zone. Dans l'océan Atlantique, la présence de ces deux espèces a été confirmée par plusieurs auteurs dont Freitas \& Biscoito (2002) et Ly (2009). D'autres prédateurs de grande taille sont aussi rencontrés dans la réserve et ils font l'objet d'une exploitation commerciale dans la zone non protégée (Epinephelus aeneus, Sphyraena afra, Lichia amia, Dasyatis margarita), mais leur abondance est relativement faible.

Une proportion d'abondance élevée de Sardinella maderensis (40,08 \%) a été notée hors de l'AMP. Par contre, son occurrence demeure relativement faible avec 14,29\%.S. maderensis est une espèce de Clupeidae, 
grégaire et de catégorie marine estuarienne, qui fréquente les estuaires lors des phases larvaires et juvéniles (Gning, 2008). La capture d'un banc de poissons peut expliquer ce résultat vu que cette espèce n'a été rencontrée que dans une seule station.

L'AFC sur les peuplements montre un peuplement dont la presque totalité des espèces sont recensées dans la réserve.Cependant, l'analyse comparée entre les deux zones a mis en évidence des abondances et biomasses plus élevées des espèces continentales (Es et Em) dans la zone protégée. Par ailleurs, l'étude des structures trophiques confirment que les espèces marines, surtout celles strictes et de haut niveau trophique, sont plus abondantes hors de l'AMP. Un résultat similaire a été obtenu par Friedlander et al. (2003) qui ont montré une biomasse $(65,8 \%)$ et un nombre total d'individus observés $(44,9 \%)$ plus importante d'herbivores dans la zone protégée de l'archipel hawaïen. Ils ont expliqué cette différence par l'habitat qu'occupent les espèces vivant dans la réserve. À Mayotte, Letourneur (1996) a également observé une composition spécifique, différente entre la réserve et la zone protégée. La zone côtière de l'AMP de Joal-Fadiouth avec la présence d'habitats, les mangroves, les fonds sableux, les fonds vaseux et l'estuaire peuvent expliquer le développement important d'espèces herbivores et de bas niveau trophique. De même, la connectivité des deux zones et la mobilité des espèces peuvent favoriser le déplacement des espèces de haut niveau trophique vers la zone non protégée comme Priacanthus arenatus et Sphyraena afra.

L'analyse de la structure en taille de la réserve montre surtout la présence des populations d'espèces de petite taille. En proportion, on trouve plus d'espèces de grande taille hors de l'AMP. L'interdiction de pêcher devait laisser normalement la possibilité aux individus de croître, ce qui a pour conséquence une augmentation de $28 \%$ de la taille moyenne des organismes (Lester et al., 2009). Par exemple dans la Box de Trévose, une réserve de pêche partielle et saisonnière située en Mer Celtique, certaines observations ont montré que la taille moyenne des espèces diminuait du centre vers l'extérieur de l'AMP. Dans cette étude, les résultats des structures de taille montrent plutôt que l'AMP est plus favorable au développement d'espèces de petite taille. Plusieurs facteurs peuvent expliquer cette situation: (i) une mauvaise surveillance contre les engins ciblant les espèces de grande taille, (ii) un milieu qui n'est pas propice à la prolifération d'espèces de grande taille et de haut niveau trophique et (iii) la mobilité des espèces.

Une vingtaine d'espèces observées en activité de reproduction montrent que l'AMP de Joal-Fadiouth est un lieu favorable à la reproduction. En outre, des individus sont rencontrés au stade juvénile. Leur longueur moyenne n'est pas élevée $(109,78 \mathrm{~mm}$ pour Brachydeuterus auritus, 93,6 mm pour Diplodus bellottii et 59,6 mm pour Chloroscombrus chrysurus). La réserve est aussi une zone de nourricerie pour plusieurs espèces halieutiques. 
Les zones de mangroves ou de faible profondeur sont considérées comme des zones de "nurserie" (Nagelkerken et al., 2000).

Comme cela est souvent le cas dans les écosystèmes marins côtiers, la biodiversité de l'AMP de Joal-Fadiouth est caractérisée par une richesse spécifique totale élevée. Par rapport à la zone hors de l'AMP, il ressort que l'ichtyofaune de la réserve est plus diversifiée. Les résultats peuvent s'expliquer par les conditions de vie pour les organismes à proximité des côtes (Rivierre, 2007). Pourtant, la diversité biologique dépendrait aussi pour partie du niveau de l'exploitation des ressources.

La comparaison des résultats entre les deux zones ne montre pas une augmentation significative de la richesse spécifique moyenne des espèces, de la biomasse et du niveau trophique moyenne. Or, des études scientifiques montrent des résultats significatifs sur la richesse spécifique, la biomasse et le niveau tropique moyen du fait de la protection des ressources marines (Lester et al., 2009 ; Mesnildrey et al., 2010). Par exemple, la mise en place de la Réserve Naturelle des Bouches de Bonifacio, composée de multiples zones comme l'AMP de Joal-Fadiouth, a des effets écologiques positifs. La biomasse de poissons est en moyenne six fois supérieure dans les zones de non prélèvement par rapport aux zones non protégées ou avec peu de surveillance (Mesnildrey et al., 2010).

Contrairement aux résultats sur la richesse spécifique, la biomasse et le niveau trophique moyen, l'abondance de la réserve est significativement plus élevée dans l'AMP. Parallèlement à l'AMP de Joal-Fadiouth, le Parc Marin de la Grande Barrière de Corail (Australie) est composé de multiples réserves dont la régulation va de l'interdiction de passage aux zones de libre accès. La densité des principales espèces pêchées à la ligne sur la Grande Barrière de Corail est significativement plus élevée dans les réserves intégrales que dans les zones où la pêche est autorisée. Après seulement deux ans de protection, l'abondance d'espèces emblématique dont l'intérêt halieutique est important, a notamment doublé (Mesnildrey et al., 2010). L'augmentation de l'abondance dans la réserve pourrait s'expliquer par l'effet de la protection qui indurait un développement des espèces herbivores et de bas niveau trophique.

\section{Conclusion}

Les différents indicateurs des peuplements de poissons analysés dans l'étude de l'efficacité bioécologique de l'AMP de Joal-Fadiouth ne répondent pas tous à l'attente exprimée lors de sa mise en place. En effet, un seul d'entre eux apporte une information significative et positive sur la mise en défens. Il s'agit de l'abondance. Par contre, l'analyse de la richesse spécifique, de la densité de biomasse et des niveaux trophiques moyens n'est pas significativement différente entre les deux zones. De plus, l'analyse des guildes fonctionnelles et des structures de taille ne dégage pas d'effets positifs 
sur les peuplements de poissons de grande taille après la mise en défens. Enfin, l'AMP se caractérise par un grand nombre de poissons herbivores, de petite taille moyenne et/ou de bas niveau trophique.

Dans l'ensemble, pour la conservation marine à petite échelle, cette étude montre des résultats positifs de la protection marine. Néanmoins, même si l'AMP bénéficie d'assez bonnes mesures de protection contre les activités anthropiques, nous avons observé une rareté des poissons de grande taille dans la zone protégée. Nous recommandons d'abord l'amélioration de la surveillance de l'AMP et la réglementation des opérations de pêche autour de celle-ci, ensuite le contrôle les captures faites dans la zone partiellement fermée et enfin la mise en place de récifs artificiels pour restaurer les habitats dégradés de certaines espèces de poissons. Il semble intéressant dans un premier temps d'étudier les causes réelles qui ont pour conséquence la prolifération des populations de petites tailles. De même, un travail sur le régime alimentaire des espèces halieutiques dans la réserve permettra de comprendre le fonctionnement de l'écosystème aquatique.

\section{References:}

1. Albaret, J. J. (1994). Les poissons : biologie et peuplements. In J.R. Durand, P. Dufour, D. Guiral, \& S.G. Zabi (Eds.), Environnement et ressources aquatiques de Côte d'Ivoire, Tome 2 : les milieux saumâtres : l'exemple de la lagune Ebrié. 239-279.Paris : ORSTOM.

2. Albaret, J.J., Simier, M. \& Sadio, O. (2005).Suivi biologique des peuplements de poissons d'une aire protégée en zone de mangrove: le bolon de Bamboung (Sine Saloum, Sénégal). Rapport final. Rapport IRD.80p.

3. Badts, V. \& Bertrand, J. (2002). Guide de la mensuration des espèces en halieutique, poissons, mollusques, crustacés, reptiles marins, mammifères marins. $18 \mathrm{p}$.

4. Claudet, J. (1991).Aires marines protégées et récifs artificiels: méthodes d'évaluation, protocoles expérimentaux et indicateurs. Doctoral dissertation, Université de Perpignan, Perpignan. 266p. 2006

5. CRODT. (1991). Situation des ressources halieutiques en 1991 et recommandations en matière de gestion. Document interne non publié, CRODT. $10 p$.

6. Diallo, A., Sène, C., Montéro, R., Diallo, A. \& Diouf, P.S. (2008).Situation de référence de la gouvernance des aires marines protégées de Joal-Fadiouth, Cacheu, Santa lucia, Branco et Rayo. Rapport REPAO. 26p.

7. Fall, M. (2009). Pêcherie démersale côtière au Sénégal: essai de modélisation de la dynamique de l'exploitation des stocks. Doctoral dissertation, Université de Montpellier 2, Montpellier. 215p. 
8. Freitas, M. \& Biscoito, M. (2002). First record of Aluterus scriptus and Aluterus monoceros (Pisces, Tetraodontiformes, Monacanthidae) from the archipelagos of Madeira and Selvagens (NE Atlantic), Museu Municipal do Funchal (História Natural), 206, 1-7. 2002. En ligne: http://dspace.cm-funchal.pt/handle/100/1574

9. Friedlander, A.M., Brown, E.K., Jokiel, P.L., Smith, W.R. \& Rodgers, K.S.(2003).Effects of habitat, wave exposure, and marine protected area status on coral reef fish assemblages in the Hawaiian archipelago. Coral Reefs, 22, 291-305. 2003. doi: 10.1007/s00338-003-0317-2

10. Gascuel, D., Laurans, M., Sidibé, A. \& Barry, M.D. (2002).Diagnostic comparatif de l'état des stocks et évolutions d'abondance des ressources démersales dans les pays de la CSRP. In Pêcheries maritimes, écosystèmes et sociétés en Afrique de l'Ouest: un demisiècle de changement. Coll. Rap. Actes du Symposium international, Dakar. 205-222.

11. Gning, C N. (2008). Ecologie trophique des juvéniles de quatre espèces de poissons dans l'estuaire inverse du Sine-Saloum (Sénégal): influence des conditions de salinité contrastées. Doctoral dissertation, Université de Montpellier 2, Montpellier. 171p.

12. Hilborn, R. (2014). Introduction to Marine Managed Areas. Advances in Marine Biology, 69, 1-13. 2014. doi: 10.1016/B978-0-12-8002148.00001-3

13. Lester,S.E., Halpern, B.S., Grorud-Colvert, K., Lubchenco, J., Ruttenberg, B.I., Gaines, S.D., \&Warner, R.R. (2009). Biological effects within no-take marine reserves: a global synthesis. Marine Ecology Progress Series, 384, 33-46. 2009. doi: 10.3354/meps08029

14. Letourneur, Y. (1996).Réponse des peuplements et populations de poissons aux réserves marines : le cas de l'île de Mayotte, Océan Indien occidental. Ecoscience, 3, 442-450. 1996doi: 10.1080/11956860.1996.11682362

15. Lobry, J. (2004).Quel référentiel de fonctionnement pour les écosystèmes estuariens?. Le cas des cortèges de poissons fréquentant l'estuaire de la Gironde. Thèse, Université de Bordeaux I, Bordeaux. $158 \mathrm{p}$.

16. Ly, A. (2009).Fonctionnement écologique et évolution du contexte socio-économique de la baie de l'étoile : une contribution à l'aménagement du littoral mauritanien et au développement d'un réseau d'aires marines protégées en Afrique de l'Ouest. Doctoral dissertation, Muséum national d'histoire naturelle de Paris, Paris. $328 \mathrm{p}$.

17. McClanahan, T.R.\& Mangi, S.C. (2004). Gear-based management of a tropical artisanal fishery based on species selectivity and capture 
size. Fisheries Management and Ecology, 11, 51-60. 2004. doi: 10.1111/j.1365-2400.2004.00358.x

18. Mesnildrey, L., Gascuel, D., Lesueur, M. \& Le Pape, O. (2006). Analyse des effets des réserves de pêche. Rapport scientifique, Les publications du Pôle halieutique, Agrocampus OUEST, Rennes. 34p.

19. Morris, D.W. (2006). Ecology: moving to the ideal free home. Nature, 443, 645-646. 2006. doi: 10.1038/443645a

20. Nagelkerken I., Van der Velde, G., Gorissen, M.W., Meijer,G.J., Van't Hof, T. \& den Hartog, C.(2000).Importance of mangroves, seagrass beds and the shallow coral reef as a nursery for important coral reef fishes, using a visual census technique. Estuarine, Coastal and Shelf Science, 51, 31-44. 2000. doi:10.1006/ecss.2000.0617

21. Ndiaye, W., Thiaw, M., Diouf, K., Ndiaye, P., Thiaw, O.T. \& Panfili, J.(2013).Changes in population structure of the white grouper Epinephelus aeneus as a result of long-term overexploitation in Senegalese waters. African Journal of Marine Science, 35, 465-472. 2013.doi:10.2989/1814232x.2013.847495

22. Ndiaye, M. (2014).Biodiversité et structures bioécologiques de la faune ichtyologique de l'aire marine protégée de Cayar (Sénégal). Université Polytechnique de Bobo-Dioulasso, Bobo-Dioulasso. 67p. 2014.

23. R Development Core Team.R: A Language and Environment for Statistical Computing. R Foundation for Statistical Computing, Vienna, Austria. ISBN 3-900051-07-0. 2015. En ligne: http://www.Rproject.org/.

24. Rivierre, A. (2007). Impacts des changements climatiques et de la pêche sur les peuplements bentho-pélagiques du plateau continental guyanais. IFREMER, Guyane. 22p.

25. Samoilys, M.A., Martin-Smith, K.M., Giles, B.G., Cabrera, B., Anticamara, J.A., Brunio, E.O. \& Vincent, A.C. (2007). Effectiveness of five small Philippines' coral reef reserves for fish populations depends on site-specific factors, particularly enforcement history. Biological conservation, 136, 584-601. doi: 10.1016/j.biocon.2007.01.003

26. Sarré, A., Fall,M., Ndour, C., \& Deme, L. (2009). Caractérisation de l'Etat de Référence des Aires Marines Protégées de Joal-Fadiouth, Kayar et Saint-Louis. CRODT, Dakar. 14p.

27. Shin, Y.J., Rochet, M.J., Jennings, S., Field, J.G. \& Gislason, H. (2005).Using size-based indicators to evaluate the ecosystem effects of fishing. ICES Journal of Marine Science: Journal du Conseil, 62, 384-396. 2005. doi: 10.1016/j.icesjms.2005.01.004 
28. Tessier, E. (2005). Dynamique des peuplements ichtyologiques associés aux récifs artificiels à l'île de la Réunion (ouest de l'océan Indien)-Implication dans la gestion des pêcheries côtières. Thèse de doctorat, Université de la Réunion, Réunion. 236p.

29. Thiam, N., Fall, M., Diadhiou, H.D., Diallo, A., Ndour, C., Sylla, M., Deme, L. \& Ndour, I. (2012). Réalisation des états de référence des zones de pêche protégées et suivis des récifs artificiels dans la zone du projet gestion durable des ressources halieutiques. Rapport final WWFWAMPO, Dakar. 78p.

30. Thiao, D., Chaboud, C., Samba, A., Laloë, F. \& Cury, P.M. (2012). Economic dimension of the collapse of the 'false cod' Epinephelus aeneus in a context of ineffective management of the small-scale fisheries in Senegal. African Journal of Marine Science, 34, 305311.doi: 10.2989/1814232X.2012.725278

31. Thiao, D. \& Mbaye, A.(2002).Évaluer et suivre les impacts des AMP sur la base d'indicateurs bio-logiques, socio-économiques et de gouvernance : Étude de cas sur l'AMP de Joal-Fadiouth. Rapport technique, USAID, Sénégal. 36p.

32. Thiaw, M., Gascuel, D., Jouffre, D. \& Thiaw, O.T. (2009).A surplus production model including environmental effects: Application to the Senegalese white shrimp stocks. Progress in Oceanography, 83, 351360. 2009.doi: 10.1016/j.pocean.2009.07.024 